\section{Case Reports in Ophthalmology}

Case Rep Ophthalmol 2020;11:364-369

DOI: $10.1159 / 000508411$
Published online: July 14, 2020

(C) 2020 The Author(s)

Published by S. Karger AG, Basel www.karger.com/cop

This article is licensed under the Creative Commons Attribution-NonCommercial 4.0 International License (CC BY-NC) (http://www.karger.com/Services/OpenAccessLicense). Usage and distribution for commercial purposes requires written permission.

\title{
Optical Coherence Tomography Angiography Findings in Optic Nerve Sheath Meningioma
}

\author{
Emanuela Interlandi ${ }^{a}$ Francesco Pellegrini $^{b} \quad$ Alessandro Papayannis $^{c}$ \\ Alessandra Cuna $^{d}$ Erika Mandarà $^{d}$ Marco De Luca ${ }^{a}$ Daniele Cirone ${ }^{e}$ \\ Cristina Ciabattoni $^{f} \quad$ Michele Marullo $^{b}$ \\ aDepartment of Ophthalmology, "Ospedale del Mare", Naples, Italy; bepartment of \\ Ophthalmology, "Santo Spirito" Hospital, Pescara, Italy; 'Department of Ophthalmology, \\ "Monfalcone-Gorizia ASUGI", Monfalcone, Italy; dDepartment of Ophthalmology, "De \\ Gironcoli" Hospital, Conegliano, Italy; eDepartment of Ophthalmology, "Villa Anna" \\ Hospital, San Benedetto del Tronto, Italy; fDepartment of Ophthalmology, "A. Murri" \\ Hospital, Fermo, Italy
}

\section{Keywords}

Optic nerve sheath meningioma - Optical coherence tomography angiography · Flow voids

\begin{abstract}
A 46-year-old female with a history of 5 months of progressive painless visual loss in the left eye was found to have an optic nerve sheath meningioma. Optical coherence tomography angiography showed a reduction in the superficial capillary plexus density consistent with her visual field defect and peripapillary retinal nerve fiber layer thinning. Moreover, abnormalities in the choriocapillaris were found in the affected eye compared to the fellow eye. Possible explanations for these findings are discussed. Further studies and a consistent number of cases are needed to correctly assess the impairment of ocular blood flow in optic nerve sheath meningioma.

2020 The Author(s) Published by S. Karger AG, Basel
\end{abstract}




\section{Case Reports in Ophthalmology}

\section{Introduction}

Optical coherence tomography angiography (OCTA) is mainly used for the study of retinal diseases but it is being recognized as a useful tool in assessing the vascular alterations of optic disc and retina in optic neuropathies. We report a case of a patient affected by optic nerve sheath meningioma (ONSM) studied by means of OCTA.

Possible explanations for OCTA findings are discussed. To our knowledge this is the first reported case of ONSM studied by means of OCTA in the English ophthalmic literature.

\section{Case Presentation}

A 46-year-old female presented complaining of progressive painless visual loss in the left eye (OS) for the previous 5 months. Best corrected visual acuity (BCVA) was 20/20 in the right eye (OD) and 20/40 OS. There was a left relative afferent pupillary defect and a 2-mm left proptosis associated with a left optic disc edema (Fig. 1 top). Standardized computerized perimetry was unremarkable OD, while it showed a peripheral constriction associated with inferior altitudinal hemianopia OS (Fig. 1 middle). The clinical presentation was consistent with a compressive optic neuropathy, confirmed by brain MRI, which showed a left ONSM (Fig. 1 bottom). OCT and OCTA of the optic nerve and retina were performed in both eyes. OCT showed a reduction of peripapillary retinal nerve fiber layer (pRNFL) consistent with the visual field defect being much denser inferiorly (Fig. 2; Fig. 3h) as well as OCTA showing a reduction of the flow at the retinal superficial capillary plexus OS (Fig. 3e) and densiometric map (Fig. 3g), particularly in the superior retina. Moreover, when compared to unaffected OD (Fig. 3a-c), OCTA interestingly showed an abnormal blood flow OS with several patches of flow voids at the level of the choriocapillaris OS (Fig. 3f).

\section{Discussion}

Meningiomas of the optic nerve represent the most common tumors of the optic nerve sheath and are characterized by the triad of long-standing poor vision, acquired optociliary shunts, and late optic atrophy [1]. ONSMs may originate from the optic nerve sheath or extend secondarily from an intracranial site. A review by Dutton [1] found primary ONSMs to represent $1.3 \%$ (22 of 1,723) of all meningiomas with an identifiable site of origin. The presence of optociliary shunts in ONSM, as a chronic sign of the disease, is well known; they represent collateral vessels connecting the choroidal and the retinal vasculature [2]. In our patient, optociliary shunt vessels were not seen because of the moderate edema and the short course of the disease. Optociliary shunt vessels are nonspecific and are seen in only $30 \%$ of patients with ONSM because they can be observed in any condition creating chronic retinal venous outflow resistance [2]. In ONSM, this resistance is caused by optic nerve compression by the surrounding tumor and optic disc edema [2]. Muci-Mendoza et al. [3] found an inverse relationship between the degree of optic disc edema and the development and easiness of visualization of the optociliary veins and their draining course through the choroidal circulation. Fluorescein angiography is unable to visualize the choriocapillaris due to absorption of the excitation wavelength by the RPE and the diffuse leakage of fluorescein from the fenestrated choriocapillaris [4]. Instead, indocyanine green angiography is the only available technique to study in vivo choroidal flow, with the limitation regarding flow velocity and flow dynamics. In 


\section{Case Reports in Ophthalmology}

Case Rep Ophthalmol 2020;11:364-369 DOI: $10.1159 / 000508411$

C 2020 The Author(s). Published by S. Karger AG, Basel www.karger.com/cop

Interlandi et al.: OCTA Findings in Optic Nerve Sheath Meningioma

addition, laser Doppler flowmetry, able to quantify flow velocity in the choriocapillaris, has the counterpart of being strictly limited to the fovea region [5]. OCTA provides an en face representation of the retina and choroid at specific depths and represents a novel and useful tool in assessing blood flow of the optic nerve head, retina, and choriocapillaris [6, 7]. Although OCTA is maximally used for the study of retinal diseases, it is being recognized as a useful tool in assessing the vascular alterations of the optic disc and retina in cases of optic neuropathies. A previous study [8] showed that a decrease in the peripapillary capillary density on OCTA corresponds to the RNFL thinning seen on standard OCT in cases of chronic optic neuropathies. Our case supports this finding since the superficial capillary plexus density reduction corresponded to the pRNFL thinning and visual field impairment. Moreover, in our patient, OCTA showed abnormal choriocapillaris blood flow OS (Fig. 4). Intercapillary spaces, identified as flow voids, have been previously reported also in unaffected eyes, particularly inferonasally and temporally, when analyzed by OCTA Swept Source (wavelength of 1,050 nm) [9]. In our opinion, the presence of flow voids in the affected eye of our patient is suggestive of a pathologic effect of the tumor rather than an incidental normal finding. The laterality, location, and dimension of the flow voids support this hypothesis. We suggest that abnormal choriocapillaris flow in our case may be due to focal arterial hypoperfusion at the level of posterior ciliary arteries, constricted by the enlarged neoplastic nerve sheaths, in particular where they enter the posterior pole at the level of the peripapillary macular region. Another possible explanation could be the rheologic effect of subacute compression of the mass on the venous return, with subsequent venostasis at the level of the choriocapillaris as a possible reason of patches of flow void seen on OCTA. Unfortunately, to date OCTA is not able to distinguish arterial from venous flow.

To the best of our knowledge, this is the first case report describing OCTA findings in ONSM. Further studies with a consistent number of cases are needed to better assess the role of OCTA in the diagnosis of optic nerve tumors.

\section{Statement of Ethics}

The patient involved in the present study gave her written informed consent to publish the case (including publication of images).

\section{Conflict of Interest Statement}

The authors have no conflicts of interest to disclose.

\section{Funding Sources}

The present study was carried out with no funding.

\section{Author Contributions}

E.I. and F.P., as first authors, accomplished all criteria for authorship recommended by ICJME, making substantial contributions to the conception and design of the work, acquisition, 


\section{Case Reports in Ophthalmology}

Case Rep Ophthalmol 2020;11:364-369

(c) 2020 The Author(s). Published by S. Karger AG, Basel www.karger.com/cop

Interlandi et al.: OCTA Findings in Optic Nerve Sheath Meningioma

analysis, and interpretation of data, and final revision of the paper. A.P., A.C., and E.M. contributed to the acquisition, analysis, and interpretation of data and final revision of the paper. M.D.L., D.C., C.C., and MM made a significant contribution to the design of the work and final revision of the paper.

All authors approved the submitted version and have agreed both to be personally accountable for the author's own contributions and to ensure that questions related to the accuracy or integrity of any part of the work, even those in which the author was not personally involved, are appropriately investigated, resolved, and the resolution documented in the literature.

\section{References}

1 Dutton JJ. Optic nerve sheath meningiomas. Surv Ophthalmol. 1992 Nov-Dec;37(3):167-83.

2 Eggers HM, Sanders MD. Acquired optociliary shunt vessels in papilloedema. Br J Ophthalmol. 1980 Apr;64(4):267-71.

3 Muci-Mendoza R, Arevalo JF, Ramella M, Fuenmayor-Rivera D, Karam E, Cardenas PL, et al. Optociliary veins in optic nerve sheath meningioma. Indocyanine green videoangiography findings. Ophthalmology. 1999 Feb;106(2):311-8.

4 Cohen SM, Shen JH, Smiddy WE. Laser energy and dye fluorescence transmission through blood in vitro. Am J Ophthalmol. 1995 Apr;119(4):452-7.

5 Urs R, Ketterling JA, Yu AC, Lloyd HO, Yiu BY, Silverman RH. Ultrasound Imaging and Measurement of Choroidal Blood Flow. Transl Vis Sci Technol. 2018 Sep;7(5):5.

$6 \mathrm{Yu} \mathrm{S,} \mathrm{Lu} \mathrm{J,} \mathrm{Cao} \mathrm{D,} \mathrm{Liu} \mathrm{R,} \mathrm{Liu} \mathrm{B,} \mathrm{Li} \mathrm{T,} \mathrm{et} \mathrm{al.} \mathrm{The} \mathrm{role} \mathrm{of} \mathrm{optical} \mathrm{coherence} \mathrm{tomography} \mathrm{angiography} \mathrm{in} \mathrm{fundus}$ vascular abnormalities. BMC Ophthalmol. 2016 Jul;16(1):107.

7 Jia Y, Morrison JC, Tokayer J, Tan O, Lombardi L, Baumann B, et al. Quantitative OCT angiography of optic nerve head blood flow. Biomed Opt Express. 2012 Dec;3(12):3127-37.

8 Chen JJ, AbouChehade JE, Iezzi R Jr, Leavitta JA, Kardon RH. Optical Coherence Angiographic Demonstration of Retinal Changes from Chronic Optic Neuropathies. Neuroophthalmology. 2017 Jan;41(2):76-83.

9 Zhang Q, Zheng F, Motulsky EH, Gregori G, Chu Z, Chen CL, et al. A Novel Strategy for Quantifying Choriocapillaris Flow Voids Using Swept-Source OCT Angiography. Invest Ophthalmol Vis Sci. 2018 Jan;59(1):203-11.

Emanuela Interlandi and Francesco Pellegrini contributed equally to this paper as first authors 


\section{Case Reports in Ophthalmology}
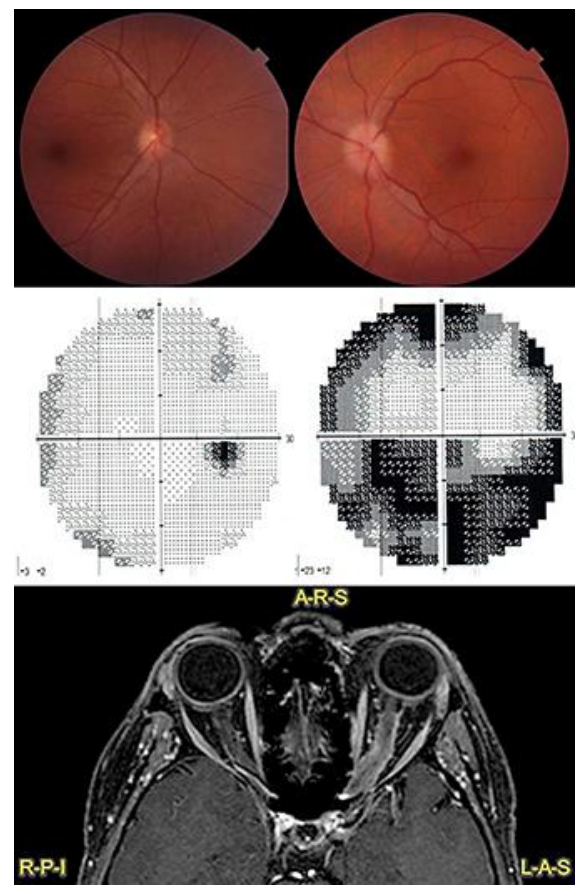

Fig. 1. Top. Dilated fundus examination demonstrates mild disc edema in the left eye; the right eye is unaffected. The left optic disc at presentation showing elevation, more pronounced nasally, superiorly, and inferiorly. Middle. Static computerized perimetry 30/2 at presentation was unremarkable OD, while it showed marked inferior altitudinal defect OS. Bottom. Axial brain T1-weighted magnetic resonance imaging showing left optic nerve sheath meningioma.
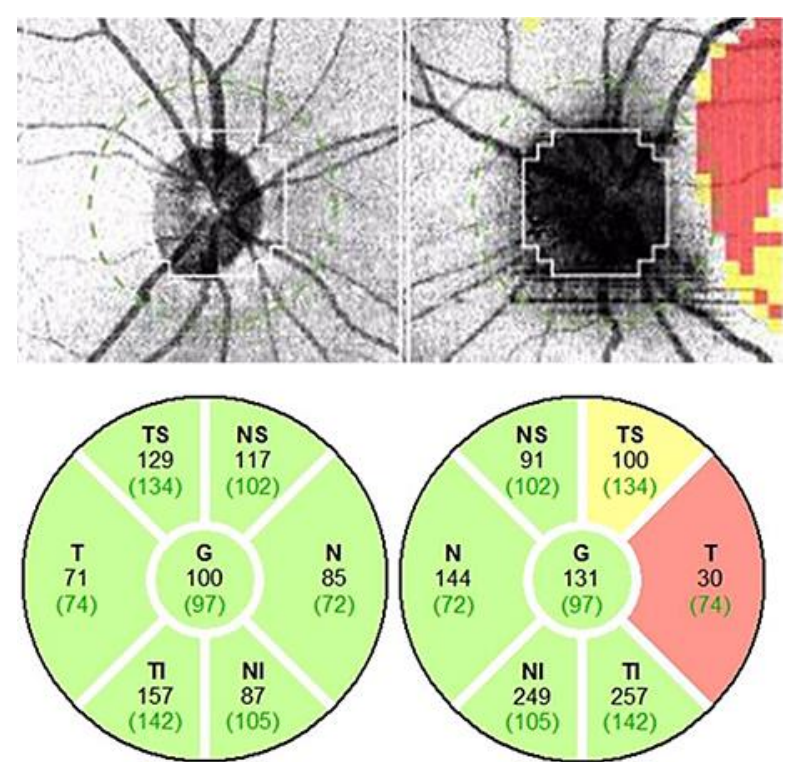

Fig. 2. Reduced thickness of the pRNFL in the temporal and superotemporal quadrants OS. OD is normal. 


\section{Case Reports in Ophthalmology}
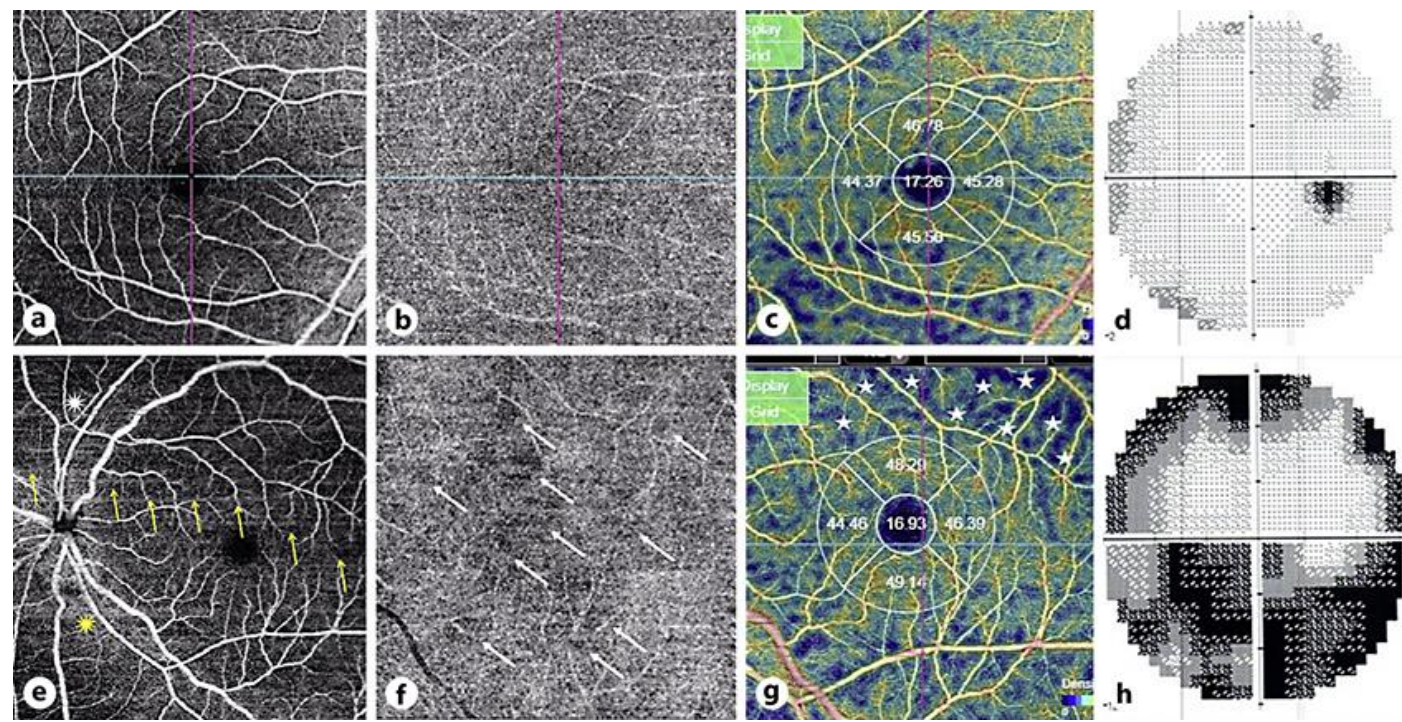

Fig. 3. a-d OCTA (macula $9 \mathrm{~mm}$ ) and perimetry OD showing no abnormalities. OCTA OS shows hypoperfusion in the superior hemiretina (white star) compared with the inferior hemiretina (yellow star) at the level of the superficial capillary plexus (arrows) (e) and multiple flow voids at the level of the choriocapillaris (f) consistent with alterations of densitometry map (white stars) (g) and pRNFL thinning and visual field defect (h). 